\title{
RESEARCH
}

Open Access

\section{Muramyl dipeptide-mediated immunomodulation on monocyte subsets exerts therapeutic effects in a mouse model of Alzheimer's disease}

Adham Fani Maleki', Giulia Cisbani', Marie-Michèle Plante ${ }^{1}$, Paul Préfontaine ${ }^{1}$, Nataly Laflamme ${ }^{1}$, Jean Gosselin ${ }^{2}$ and Serge Rivest ${ }^{1 *}$

\begin{abstract}
Background: Muramyl dipeptide (MDP) is a component derived from minimal peptidoglycan motif from bacteria, and it is a ligand for the NOD2 receptor. Peripheral administration of MDP converts Ly6C ${ }^{\text {high }}$ into Ly6Clow monocytes. Previously, we have shown that Ly6 $\mathrm{C}^{\text {low }}$ monocytes play crucial roles in the pathology of a mouse model of Alzheimer's disease (AD). However, medications with mild immunomodulatory effects that solely target specific monocyte subsets, without triggering microglial activation, are rare.

Methods: Three months old APP swe/PS1 transgenic male mice and age-matched C57BL/6 J mice were used for high frequency (2 times/week) over 6 months and low frequency (once a week) over 3 months of intraperitoneally MDP (10 $\mathrm{mg} / \mathrm{kg}$ ) administrations. Flow cytometry analysis of monocyte subsets in blood, and behavioral and postmortem analyses were performed.

Results: Memory tests showed mild to a strong improvement in memory function, increased expression levels of postsynaptic density protein 95 (PSD95), and low-density lipoprotein receptor-related protein 1 (LRP1), which are involved in synaptic plasticity and amyloid-beta $(A \beta)$ elimination, respectively. In addition, we found monocyte chemoattractant protein-1(MCP-1) levels significantly increased, whereas intercellular adhesion molecule-1(ICAM1) significantly decreased, and microglial marker (Iba1) did not change in the treatment group compared to the control. In parallel, we discovered elevated cyclooxygenase-2 (COX2) expression levels in the treated group, which might be a positive factor for synaptic activity.

Conclusions: Our results demonstrate that MDP is beneficial in both the early phase and, to some extent, later phases of the pathology in the mouse model of AD. These data open the way for potential MDP-based medications for AD.
\end{abstract}

Keywords: NOD2 receptor, Monocytes, Microglia, Macrophages, Alzheimer's disease, Immunotherapy, Brain blood vessels, Cerebral amyloid angiopathy, Synapse

\footnotetext{
* Correspondence: serge.rivest@crchudequebec.ulaval.ca

'Neuroscience Laboratory, CHU de Quebec Research Center and Department of Molecular Medicine, Faculty of Medicine, Laval University, 2705 Laurier Boulevard, Quebec City, QC G1V 4G2, Canada

Full list of author information is available at the end of the article
}

(c) The Author(s). 2020 Open Access This article is licensed under a Creative Commons Attribution 4.0 International License, which permits use, sharing, adaptation, distribution and reproduction in any medium or format, as long as you give appropriate credit to the original author(s) and the source, provide a link to the Creative Commons licence, and indicate if changes were made. The images or other third party material in this article are included in the article's Creative Commons licence, unless indicated otherwise in a credit line to the material. If material is not included in the article's Creative Commons licence and your intended use is not permitted by statutory regulation or exceeds the permitted use, you will need to obtain permission directly from the copyright holder. To view a copy of this licence, visit http://creativecommons.org/licenses/by/4.0/ The Creative Commons Public Domain Dedication waiver (http://creativecommons.org/publicdomain/zero/1.0/) applies to the data made available in this article, unless otherwise stated in a credit line to the data. 


\section{Background}

MDP is derived from minimal bioactive peptidoglycan motif from most Gram-negative and -positive bacteria [1] and mediates its effects on the immune response via NOD2 receptor $[1,2]$. This receptor is a member of the NLR family of leucine-rich repeat proteins [1] and is strongly expressed in monocyte precursors that have the ability to differentiate into pro-inflammatory and patrolling subsets and macrophages once infiltrating tissues [3].

In humans, monocyte subsets are characterized by expression levels of CD14 and CD16, as being, classical $\left(\mathrm{CD} 14^{++} \mathrm{CD} 16^{-}\right)$, intermediate $\left(\mathrm{CD} 14^{++} \mathrm{CD} 16^{+}\right)$, and non-classical $\left(\mathrm{CD} 14^{+} \mathrm{CD} 16^{++}\right)$subsets [4]. In mice, proinflammatory monocytes are characterized by a combination of surface markers (CX3CR $1^{\text {low } C C R 2} 2^{+}$Ly $6 C^{\text {high }}$ ), whereas patrolling monocytes are defined as

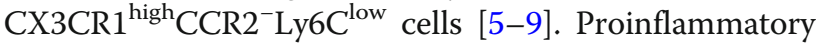
monocytes are involved in inflammatory responses, extravasate in inflamed tissues in a CCR2-dependent manner, and thus contribute to local inflammation. On the other hand, patrolling monocytes (also referred to as anti-inflammatory) establish the resident regulatory patrolling monocyte population [10]. Ly6 $\mathrm{C}^{\text {low }}$ monocytes are resident phagocytes that patrol the lumen of blood vessels and enhance tissue repair [10].

$\mathrm{AD}$ is characterized by the chronic activation of innate immune cells within the CNS. AD is associated with $A \beta$ accumulation in the parenchyma and cerebral vasculature due to impaired clearance of the neurotoxic $A \beta_{1-40}$ and $A \beta_{1-42}$ peptides $[11,12]$. Several lines of evidence indicate that cerebral amyloid angiopathy (CAA) acts as a significant contributor to the AD pathology [13, 14]. CAA is mainly caused by an impaired $A \beta$ clearance from the cerebral vasculature along perivascular lymphatic drainage pathways. Having more than $90 \%$ prevalence in AD patients clearly shows its significant impact on AD pathology and cognitive decline $[13,14]$.. More importantly, there is a constant equilibrium between $A \beta$ vascular/peripheral and parenchymal levels [15, 16]. Therefore, the clearance of $\mathrm{A} \beta$ in perivascular spaces reduces the burden in the parenchyma through equilibrium-driven redistribution $[16,17]$.

The blood-brain barrier (BBB) structure limits access to select soluble molecules and circulating leukocytes in the CNS [17, 18]. Among leukocytes, monocytes and monocyte-derived perivascular macrophages have a crucial role in AD. Indeed, evidence from previous studies suggests that monocyte-derived perivascular macrophages are highly efficient for $A \beta$ phagocytosis $[19,20]$. In parallel, several studies highlighted the crucial effect of Ly6C $\mathrm{C}^{\text {low }}$ monocyte subset in AD. There is a reduction in non-classical $\mathrm{CD} 14^{+} \mathrm{CD} 16^{++}$monocytes in $\mathrm{AD}$ patients compared with mild cognitive impairment patients or age-matched healthy controls [21]. Our group has shown that Ly $6 C^{\text {low }}$ monocytes internalize $A \beta$ and efficiently eliminate $\mathrm{A} \beta$ microaggregates and transport them from the brain microvasculature to the blood circulation in a two-photon microscopy study [22].

In this study, we investigated whether the immunomodulatory effects of MDP could influence the neuropathology of an APP mouse model of AD. We found that MDP administrations converted Ly $6 C^{\text {high }}$ into Ly6 $C^{\text {low }}$ monocytes, which was associated with improvement in memory function together with the increased expression of markers of synaptic plasticity and $A \beta$ clearance.

\section{Methods \\ Animal Care}

All protocols were performed according to the Canadian Council on Animal Care guidelines, as administered by the Laval University Animal Welfare Committee. All experiments were approved by the local committee. All efforts were made to avoid their suffering. All mice were maintained in a pure $\mathrm{C} 57 \mathrm{BL} / 6 \mathrm{~J}$ background, bred in house, and newborn pups were genotyped with PCR as advised by Jackson Laboratory protocols. All animals were housed up to four per cage in temperature and light-controlled room $(12 \mathrm{~h}$ light cycles from 7 am to 7 pm) and were fed (mouse chow) and allowed to drink water ad libitum. All mice were monitored for health status, including weight loss throughout all experimental protocols.

\section{APP model and MDP treatment}

$\mathrm{APP}_{\text {Swe }} / \mathrm{PS} 1$ expressing the chimeric mouse/human amyloid precursor protein (Mo/HuAPP695swe) and a mutant human presenilin 1 (PS1-dE9) under the control of independent mouse prion promoter elements [B6.CgTg(APPswe,PSEN1dE9)85Dbo/J]. A total of fifty-five 3 months old male APPswe/PS1 transgenic mice and twenty-five age-matched C57BL/6 J wild-type mice (WT) were utilized. Mice were injected one/two times per week with either MDP commercially available (Catalog \# tlrl-MDP, Version \# 16A22-MM, Invivo Gen) diluted in saline $(10 \mathrm{mg} / \mathrm{kg}$ ) or vehicle (saline $0.9 \%)$.

\section{Flow cytometry}

Blood samples were collected from the submandibular vein and kept in EDTA coated vials on a rotator for $<1$ $\mathrm{h}$, and fluorescence-activated cell sorting (FACS) analysis was performed as described by [23, 24]. FACS and data acquisition were performed using SORP LSR II and FACSDiva softwares (both from BD), respectively. Results were analyzed with the FlowJo software (v10.0.7). For details on flow cytometry protocol, see Additional file 1, Fig. 2 [24]. 


\section{Sacrifices}

All mice were sacrificed via intracardiac perfusion with $0.9 \%$ saline, followed by $4 \%$ PFA pH 7.4. The brains were then retrieved, post-fixed $10-24 \mathrm{~h}$ in $4 \%$ PFA pH 7.4, and transferred in $4 \%$ PFA pH $7.4+20 \%$ sucrose for a minimum of $15 \mathrm{~h}$. In another set of experiments, brains were retrieved, and one hemisphere was snap-freeze for protein extraction while the other hemisphere was fixed in $4 \%$ PFA pH $7.4+20 \%$ sucrose. Brains were sliced in coronal sections of $25-\mu \mathrm{m}$ thickness with a freezing microtome (Leica Microsystems), serially collected in an anti-freeze solution and kept at $-20^{\circ} \mathrm{C}$ until usage.

\section{Post-mortem analysis} Immunofluorescence

Brain sections were washed four times for $5 \mathrm{~min}$ in KPBS and then blocked in KPBS containing 1\% BSA, 4\% NGS, and $0.4 \%$ Triton X-100. The tissues were incubated overnight at $4{ }^{\circ} \mathrm{C}$ with the primary Iba- 1 antibody (1: 2,000; Wako Chemicals) and monoclonal anti-A $\beta$ (6E10, 1: 3000; Covance). After washing four times for 5 min in KPBS, tissues were incubated in the appropriate secondary antibody (IgG anti-mouse Alexa 488; Thermofisher and IgG anti-rabbit CY3; Jackson Immunoresearch) for $2 \mathrm{~h}$ at room temperature. Following further washes in KPBS and incubation with DAPI, the sections were mounted onto Micro Slides Superfrost Plus glass slides and coverslipped with Fluoromount-G (Electron Microscopy Sciences).

\section{Image acquisition and analyses}

Image acquisition of fluorescent staining images was performed using a Zeiss LSM800 confocal microscope supported by the Zen software (2.3 system) using the $x$ 4 and $\times 40$ lenses, as described previously [25]. The number of $6 \mathrm{E} 10$, Iba-1 associated with plaques, was quantified by unbiased stereological analysis [26] using the Stereo Investigator software (version 6.02.1, MicroBrightfield) attached to a Nikon C80i microscope equipped with a motorized stage (Ludl) attached to Microfire CCD color camera (Optronics). Four to six sections were analyzed for each animal.

\section{Soluble $A \beta_{1-42} / A \beta_{1-40} E L I S A$}

Brain levels of soluble $A \beta_{1-42}$ and $A \beta_{1-40}$ were quantified by using the Human Amyloid $\beta 42$ and Human Amyloid $\beta 40$ Brain ELISA kits (Millipore, Billerica, MA, USA). The experimental procedure was performed according to the manufacturer's instructions [27].

\section{Western blot analysis}

Hippocampus and cortex brain proteins were lysate, as previously described [27]. Proteins were then loaded in 4-15\% agarose precast gels (Biorad) and electroblotted onto $0.45 \mu \mathrm{m}$ Immobilon PVDF membranes. Membranes were immunoblotted with various primary antibodies, as described in Table 1, followed by the appropriate horseradish peroxidase (HRP)-conjugated secondary antibodies and revealed by enhanced chemiluminescence plus (ECL) solution (GE Healthcare Life Sciences). Quantification was done by determining the integrative density of the bands using the Thermo Scientific Pierce myImage Analysis Software v2.0. Optical values were normalized over actin.

\section{Behavioral tests \\ Open field}

The open field was performed to evaluate anxiety-like behaviors, exploration habits, and also locomotor activity, as described by Hui et al. [28]. Each mouse was individually recorded and analyzed by the ANY-maze system.

\section{Water $T$ maze}

The water $\mathrm{T}$ maze assay was performed, according to Guariglia et al. [29]. The pool was filled with $23^{\circ} \mathrm{C}( \pm 1$ C) water to a depth of $13 \mathrm{~cm}$, which was $1 \mathrm{~cm}$ above the surface of the platform. Mice were trained to swim to a particular arm of the $\mathrm{T}$ maze and to remain on a submerged platform for $5 \mathrm{~s}$. Mice had to complete six out of eight trials without error for two consecutive days out of three days to reach the learning criterion. The same criterion was considered for the reversal phase.

\section{Statistics}

Data are expressed as the mean \pm SEM. A comparison between two groups was conducted using post hoc unpaired $t$ tests. Comparisons between more than two treatment groups were performed using either one-way analysis of variance (ANOVA) or two-way repeatedmeasures ANOVA, followed by Tukey's post-hoc test or uncorrected Fisher's Least Significant Difference test. Values were statistically significant if $p<0.05$. All analyses were performed using GraphPad Prism Version 6 for Windows (GraphPad Software, San Diego, CA, USA) and SAS 9.4 (SAS Institute Inc., Cary, NC, USA). All panels were assembled using Adobe Photoshop CS5 (version 12.0.4) and Adobe Illustrator CS5 (version 15.0.2).

\section{Results}

Chronic and high frequency of MDP administration in the APP mouse model of AD show a slight improvement in memory function

Our team has previously demonstrated a critical role of Ly6 $C^{\text {low }}$ monocytes in $A \beta$ clearance within the cerebrovascular system. Indeed, $\mathrm{Ly} 6 \mathrm{C}^{\text {low }}$ monocytes are able to associate within $\mathrm{A} \beta$-positive veins but not arteries, 
Table 1 Represents antibodies used for immunoblotting analyses

\begin{tabular}{|c|c|c|c|c|c|c|}
\hline Antibody & Company & Molecular weight & Species & Dilution & Secondary antibody dilution & Notes \\
\hline Actin & Millipore & $42 \mathrm{kDa}$ & Mouse & $1 / 50,000$ & $1 / 20000$ & \\
\hline APP & Millipore & $\approx 100 \mathrm{kDa}$ & Mouse & $1 / 2000$ & $1 / 5000$ & $\begin{array}{l}\text { antigen retrival (Michaud, } \\
\text { Hallé et al. 2013) }\end{array}$ \\
\hline Cox-2 & Santa Cruz & $\approx 75 \mathrm{kDa}$ & Goat & $1 / 1000$ & $1 / 5000$ & \\
\hline |ba-1 & Wako & $18 \mathrm{kDa}$ & Rabbit & $1 / 1000$ & $1 / 5000$ & \\
\hline ICAM & Santa Cruz & $85-110 \mathrm{kDa}$ & Goat & $1 / 500$ & $1 / 1000$ & \\
\hline LRP1 & CEDARLANE & $85 \mathrm{kDa}$ & Rabbit & $1 / 1000$ & $1 / 40,000$ & \\
\hline MCP1 & Cell Signaling & $13 \mathrm{kDa}$ & Rabbit & $1 / 1000$ & $1 / 5000$ & \\
\hline PSD95 & Neuromab & $95 \mathrm{kDa}$ & Mouse & $1 / 2000$ & $1 / 20,000$ & \\
\hline Synaptophysin & Thermo Fisher Scientific & $34 \mathrm{kDa}$ & Mouse & $1 / 10000$ & $1 / 100,000$ & \\
\hline Trem2 & R\&D Systems & $40 \mathrm{kDa}$ & Rabbit & $1 / 500$ & $1 / 2000$ & $\begin{array}{l}\text { antigen retrival (Michaud, } \\
\text { Hallé et al. 2013) }\end{array}$ \\
\hline VCAM & Santa Cruz & 90-100 kDa & Rabbit & $1 / 1000$ & $1 / 10,000$ & \\
\hline NFkB p50 & CEDARLANE & $50 \mathrm{kDa}$ & Rabbit & $1 / 1000$ & $1 / 10,000$ & \\
\hline
\end{tabular}

Table 1 represents antibodies used for immunoblotting analyses and all related information, including the name of the company, molecular weight, species, secondary antibodies, and dilution rates

internalize $A \beta$, and efficiently eliminate $A \beta$ microaggregates and transport them from the brain microvasculature to the blood circulation [22]. Immune modulation mediated by MDP in shifting monocyte subsets towards Ly6 $\mathrm{C}^{\text {low }}$ prompted us to assess potential therapeutic effects of MDP in APP mice. We chronically administered MDP twice a week (high frequency) in 3-month-old APP mice over 6 months (Fig. 1a). We then evaluated the circulating monocyte subsets at both 3 and 6 months following the beginning of the injections. The APP mice develop an Alzheimer-like phenotype at 6 months of age. In parallel, 4-6 months old APP mice develop small and punctate $A \beta$ aggregates accumulation on specific blood vessels [22]. Thus, these time points were chosen to evaluate whether MDP is capable of delaying disease onset (3 months following the first MDP injection) and maintain the phenotype over time ( 6 months after the first MDP injection). The drug was able to modulate Ly6 $C^{\text {high }}$ monocytes towards the Ly6C ${ }^{\text {low }}$ subset at both 3- and 6-month post-injection times (Fig. 1b, c). To assess whether MDP affects cognitive behavior, we performed the water T-maze test. We observed that APP mice that received MDP did not significantly differ from their counterparts that received saline both during the learning and reversal phases of the water $\mathrm{T}$-maze test (Fig. 1d, e). Nevertheless, shifting in the percentage of mice with errorless trials in both groups at the two time points suggests a slight improvement in performing the memory test in the treatment group. Indeed, at 3 months post-injection, the percentages of mice with errorless trials were 71.4 and 50 in control and treatment groups, respectively. On the contrary, at 6 months post-injection, we observed that the control group showed a reduction in the percentage and reached $50 \%$. In contrast, the treatment group demonstrated an increase in the percentage $(87.5 \%)$ of mice with errorless trials (Fig. 1f). Moreover, analysis of the average of total errors in the reversal phase of the test between treatment and control groups at the two time points also suggests a slight improvement in memory function. More precisely, we did not find a significant change in the average of total errors between treatment and control groups in the two time points. Nonetheless, the treatment group (but not the control group) showed a tendency $(p=0.0690)$ to have a lower number of total errors at the second time point when compared with the first time point (Fig. 1g). In the open field test, the results did not show any significant difference between the group treated with saline or MDP, indicating that the treatment caused neither anxiety-like behaviors nor locomotor activity problems (data not shown). Overall, these results suggest that monocytes are modulated in APP mice, and MDP treatments slightly improved cognitive deficits of the mice when the disease is established.

\section{A chronic and low frequency of MDP administration improves cognitive deficits in the APP mouse model of AD}

We then tested whether chronic MDP injection in APP and WT mice is at a lower frequency (once a week) over 3 months (Fig. 2a). Similar to the high-frequency MDP administration, a low-frequency MDP administration was able to shift monocyte subsets towards the Ly6 $\mathrm{C}^{\text {low }}$ phenotype (Fig. 2b, c). In the water T-maze test, first, we analyzed learning curves in the training phase using a two factor (treatment $x$ day) ANOVA with repeated measures on day. We only observed a significant effect of day between groups $(F(2,48)=21.18, P<0.0001)$. 


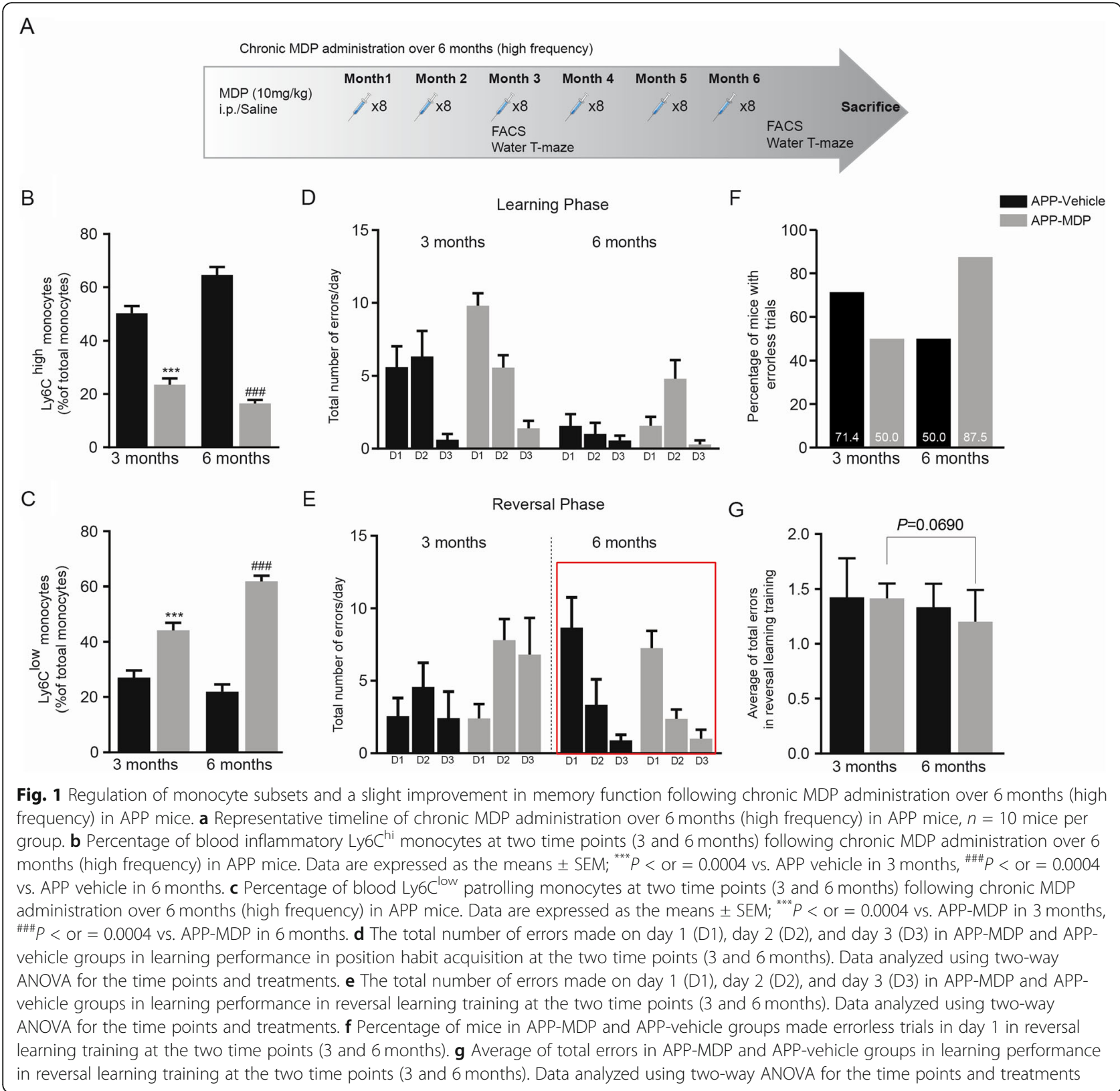

Although we observed a significant effect of day between groups, Post-hoc comparisons showed no difference for every single day when we compared all groups (even WT-vehicle versus APP-vehicle). That might be related-at least partially-to the fact that the water Tmaze has a single choice point with only two alternatives, which increases the possibilities of success (by default, the probability of choosing the correct arm is 50\%) reviewed in details by Sharma and colleagues [30]. Therefore, we performed a repeated-measures ANOVA test on intra-group performance to see whether every group during 3 days training phase was able to make statistically significantly fewer errors. Although at different rates, WT groups overall showed improvement by making fewer errors on the second and third days in comparison with the first day of the training phase (Fig. 2d). Moreover, the improvement in the WT-vehicle group reached statistical significance (Fig. 2d), whereas mice in the APP control group did not show any significant difference on the same days. More importantly, they made more errors on the third day compared with the second day, indicating that they were impaired in their ability to consolidate the entering right arm following two consecutive days of training (Fig. 2d). In 

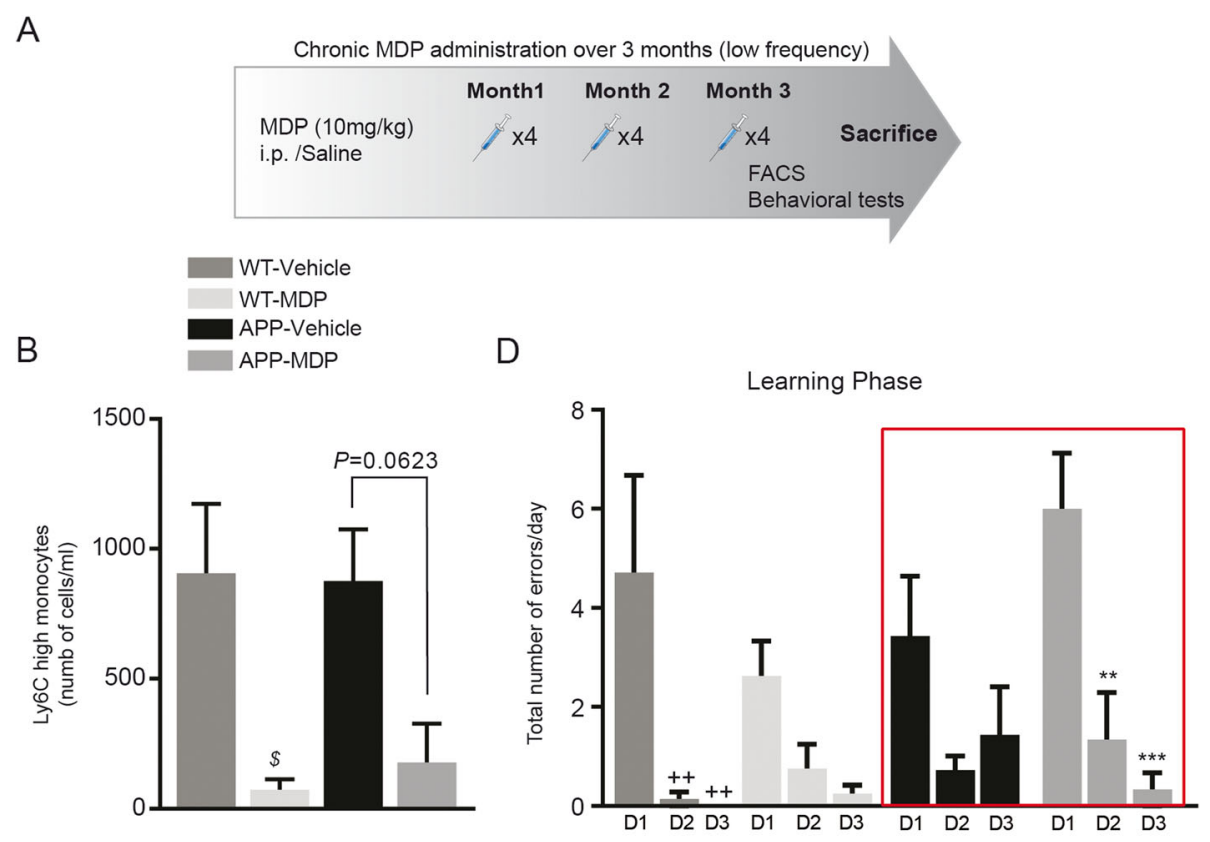

C

$E$
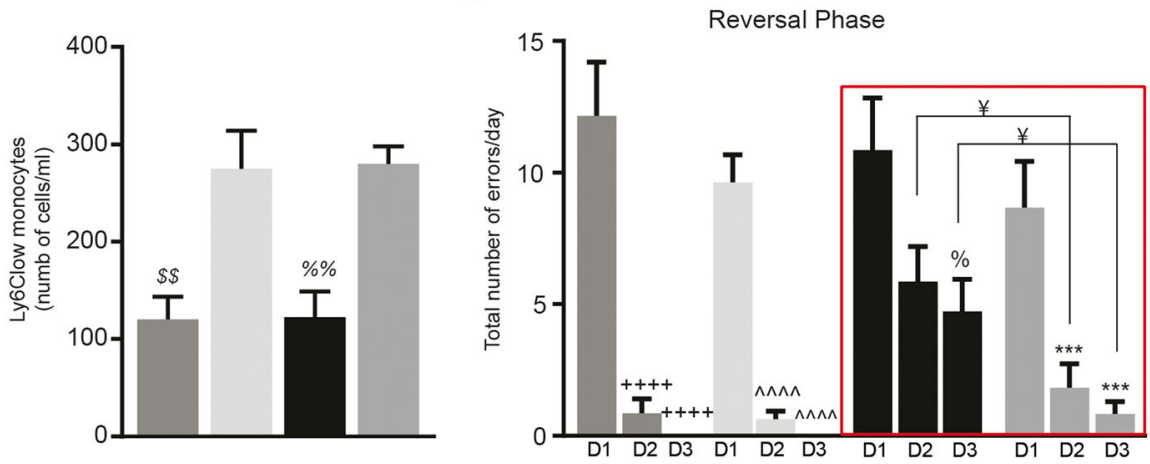

Fig. 2 Regulation of monocyte subsets and improvement in memory deficits following chronic MDP administration over 3 months (low frequency) in APP mice. a Representative timeline of chronic MDP administration over 3 months (low frequency) in APP and WT mice, APP $n=10$ mice per group, and WT $n=5$ mice per group. $\mathbf{b}, \mathbf{c}$ Absolute count of blood inflammatory Ly6C ${ }^{\text {hi }}$ monocytes in WT and APP mice and following chronic MDP administration over 3 months (low frequency). Data are expressed as the means \pm SEM; ${ }^{\$} P<$ or $=0.01 \mathrm{vs}$. WT-vehicle. $\mathbf{c}$ Absolute count of blood Ly6C ${ }^{\text {low }}$ monocytes in WT and APP mice and following chronic MDP administration over 3 months (low frequency). Data are expressed as the means $\pm \mathrm{SEM} ;{ }^{\$} \mathrm{P}<$ or $=0.003$ vs. WT-MDP, ${ }^{\%} \%$ < or $=0.007$ vs APP-MDP. $\mathbf{d}$ The total number of errors made on day 1 (D1), day 2 (D2), and day 3 (D3) in WT and APP mice in learning performance in position habit acquisition following chronic MDP administration over 3 months (low frequency). Data are expressed as the means \pm SEM; ${ }^{++} P<$ or $=0.002$ vs. WT-vehicle D1, ${ }^{* *} P<$ or $=0.003$ vs. APP-MDP D1, ${ }^{* * *} P<$ or $=0.0004$ vs APP-MDP D1. Data analyzed using two-way ANOVA for the time points and treatments. e The total number of errors made on day 1 (D1), day 2 (D2), and day 3 (D3) in WT and APP mice in reversal performance in position habit acquisition following chronic MDP administration over 3 months (low frequency). Data are expressed as the means $\pm \mathrm{SEM} ;{ }^{¥} P<$ or $=0.0266$ vs. APP-vehicle D2 and D3, ${ }^{++++} P<$ or $=0.0001$ vs. WT-vehicle D1, ${ }^{\wedge \wedge \wedge} P<$ or $=0.0001$ vs. WT-MDP D1 ${ }^{\%} P<$ or $=0.0092$ vs. APP-vehicle D1, ${ }^{* * *} P<$ or $=0.0008$ vs. APP-MDP D1. Data analyzed using two-way ANOVA for the time points and treatments

contrast, the APP treatment group made significantly fewer errors in the second and third days in comparison with the first day of training phase (Fig. 2d).

Next, using a two factor (treatment $\times$ day) ANOVA with repeated measures on day, we analyzed all groups in the reversal phase, which is the most important phase of the water T-maze test. ANOVA revealed a significant effect of treatment $(F(2,48)=73.12, P<0.0001)$ and a significant effect of day $(F(3,24)=6.006, P=0.0033)$.
Post-hoc comparisons (uncorrected Fisher's Least Significant Difference test) revealed significant differences between the control groups and the treatment group. More precisely, on the second and third days, there was a significant difference between APP control and treatment groups (Fig. 2e). Although it is not shown in the figure, we found a significant difference on the second day, in both WT groups versus APP control $(P<$ $0.0016)$, and on the third day, in both WT groups versus 
APP control $(P<0.0042)$. We also reported the results from repeated-measures ANOVA on intra-group tests for the reversal phase (Fig. 2e).

Besides the water T-maze test, we observed the same results as the high-frequency protocol from the open field test (data not shown). These results suggest that chronic administration of MDP at lower frequency is sufficient to delay the appearance of an Alzheimer-like phenotype. Collectively, the behavioral test results obtained from both protocols suggest that low-frequency MDP administration (once per week) is more effective than high-frequency administration (twice per week).

\section{MDP-derived memory improvement is not dependent on} the change in $A \beta$ levels and microglial activation

Microglial cells play a key role in AD pathogenesis by regulating $A \beta$ levels in the brain via uptake and degradation processes. Therefore, we evaluated whether MDP treatment could impact $A \beta$ accumulation and microglial functions. We measured the number of Iba1-positive microglia associated with 6E10-positive plaques as well as the number of immunostained plaques in both the hippocampus and cortex of APP mice that received MDP or saline. We did not observe any significant difference between the two groups (see Additional file 1, figure S1A- $\mathrm{S} 1 \mathrm{H})$. We subsequently assayed soluble $A \beta 40$ and $A \beta 42$ levels in cortex and hippocampus by specific ELISA immunoassays, and even in this case, the results showed no significant difference between treatment and control groups (see Additional file 1, figure S1I and S1J). We next measured several markers in both protocols. To avoid redundancy, in this section, as well as the following sections, we have presented the results only from the high-frequency protocol.
As $A \beta$ is produced through sequential cleavage of APP, catalyzed by $\beta$ - and $\gamma$-secretase [31], we measured the expression level APP by immunoblot, and we did not observe any change in APP level in both groups, (Fig. 3a). In addition, we determined the expression levels of Iba1, TREM2, and nuclear factor-kB (NF-kB, P50) in the hippocampus of APP mice treated with MDP or saline, and no differences were observed (Fig. $3 \mathrm{~b}, \mathrm{c})$. Finally, we observed significantly higher levels of COX2 in the brain of MDP-treated mice (Fig. 3d), which may be indicative of synaptic plasticity [32-34]. Altogether, these results indicate that the memory/learning improvements observed in behavioral tests are not dependent on the $A \beta$ burden or microglial activation, suggesting other factor(s) involved in MDP-mediated cognitive improvement.

\section{MDP-derived memory improvement mediated by modification of synaptic function and vascular clearance of $A \beta$}

We next asked if MDP-derived memory improvement is dependent on improvement in synapse formation. Hence, we quantified pre- and postsynaptic puncta (synaptophysin and PSD95) in treatment and control groups. Immunoblot analysis of synaptophysin showed no significant difference (Fig. 4a). However, we found that PSD95 levels significantly increased in APP-MDP compared to those of control mice (Fig. 4b). Consequently, we assessed the LRP1 level as this protein interacts and co-localizes with PSD95 for synapse formation and is a key player to eliminate $A \beta$ across the BBB [35]. Interestingly, LRP1 protein expression levels also increased significantly in the group treated with MDP (Fig. 4c). Altogether, these results indicate that memory

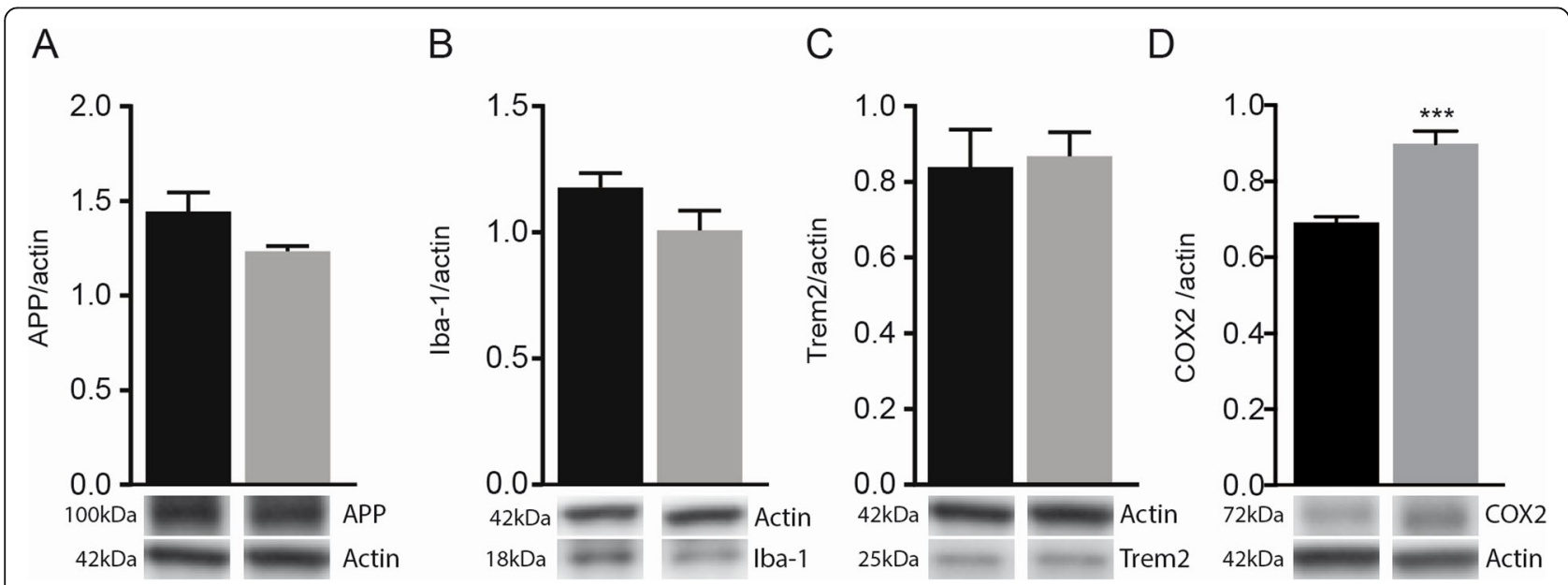

Fig. 3 MDP treatment had no effect on microglial activation, and APP levels, but upregulated COX2 levels in APP mice. a Immunoblot analysis of APP levels in the cortex and hippocampus of APP mice treated with vehicle and MDP. $\mathbf{b}, \mathbf{c}$ Immunoblot analysis of Iba1 and TREM 2 levels in the cortex and hippocampus of APP mice treated with vehicle and MDP. $\mathbf{d}$ Immunoblot analysis of COX2 levels in the cortex and hippocampus of APP mice treated with vehicle and MDP. Data are expressed as the means \pm SEM; ${ }^{* * *} P<0.0001$ vs. APP-MDP 

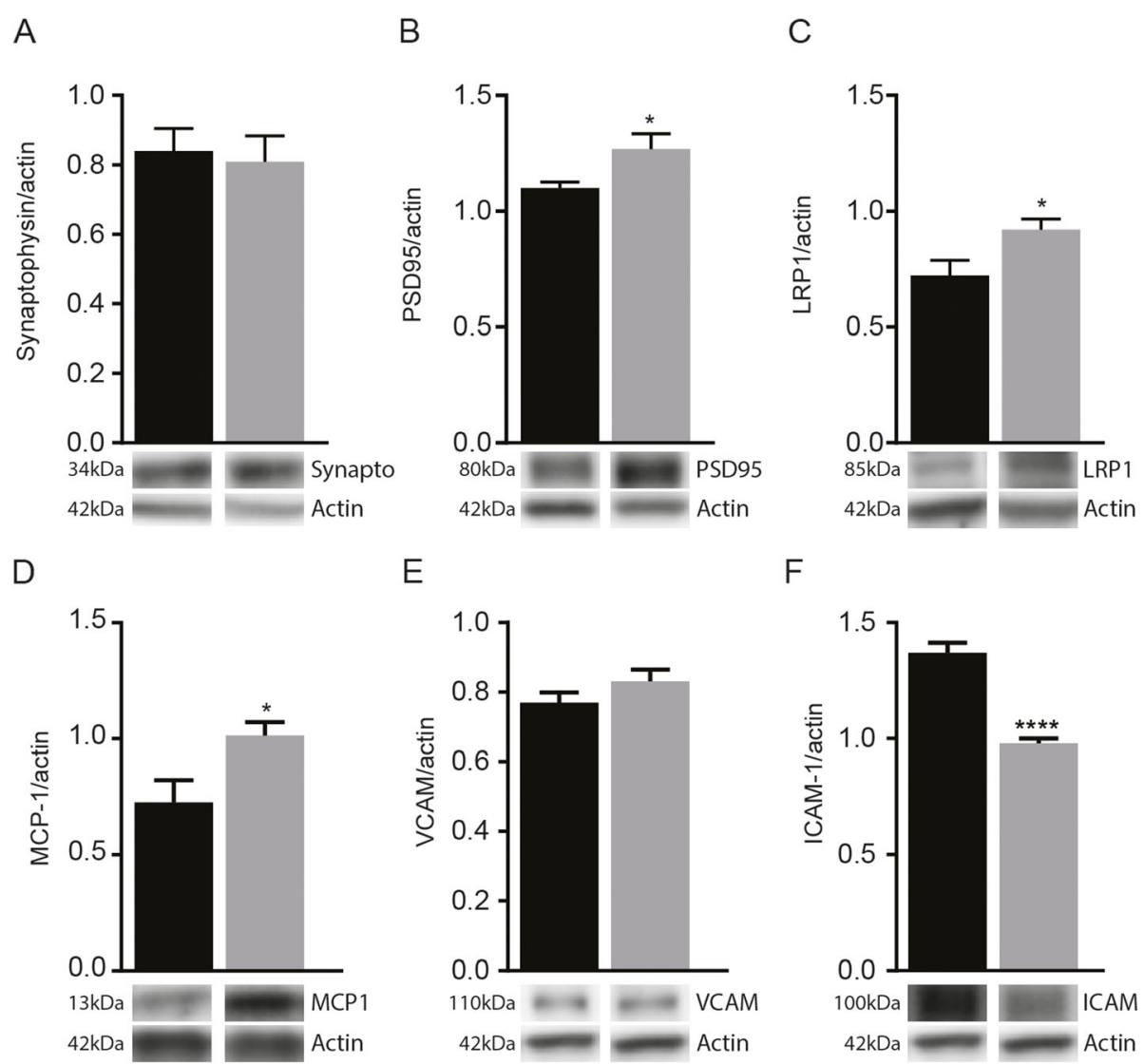

Fig. 4 Effect of MDP treatment on key proteins involved in synaptic functions, A $\beta$ vascular clearance, and cerebrovascular monocyte adhesion. a, b Immunoblot analysis of synaptophysin and PSD95 protein levels, respectively, in the cortex and hippocampus of APP mice treated with vehicle and MDP, $n=10$ mice per group. Data are expressed as the means $\pm S E M ;{ }^{*} P<$ or $=0.03$. $\mathbf{c}$, $\mathbf{d}$ Immunoblot analysis of LRP1 and MCP-1 protein levels respectively in the cortex and hippocampus of APP mice treated with vehicle and MDP, $n=10$ mice per group. Data are expressed as the means \pm SEM; ${ }^{*} P<$ or $=0.03$. e, $\mathbf{f}$ Immunoblot analysis of VCAM and ICAM-1 protein levels, respectively, in the cortex and hippocampus of APP mice treated with vehicle and MDP, $n=10$ mice per group. Data are expressed as the means \pm SEM; ${ }^{* * * *} P<$ or $=0.0001$

improvement mediated by MDP may depend on the enhancement of synaptic plasticity and vascular $A \beta$ clearance.

\section{Effect of MDP on key proteins involved in cerebrovascular monocyte adhesion}

Because we did not observe any differences at the microglial level, we evaluated whether proteins normally associated with monocyte recruitment and vascular adhesion which were modulated following MDP treatments. Monocyte chemoattractant protein-1 (MCP-1) levels play a crucial role in the recruitment monocytes along with the cerebrovascular elements, and they increased significantly in the brain of mice treated with MDP (Fig. $4 \mathrm{~d})$. Interestingly, as mentioned before, no significant change was detected for NF-kB (data not shown). We then evaluated the expression levels of vascular cell adhesion molecule-1 (VCAM-1) and intercellular adhesion molecule-1 (ICAM-1). VCAM-1 showed no significant changes, whereas a reduction in the expression level of
ICAM-1 was observed in mice treated with MDP when compared to the control group (Fig. 4e, f). Consistent with our previous observations, these results indicate that MDP has no apparent neuroinflammatory effects in the brain but can modulate the levels of chemotactic factors. Finally, the synaptic markers, LRP1, the monocyte recruitment, and vascular adhesion markers, did not show a significant change in WT-MDP when compared with WT-vehicle (data not shown).

\section{Discussion}

Previously, we have shown a critical role of Ly6C $\mathrm{C}^{\text {low }}$ monocytes in the cerebrovascular $\mathrm{A} \beta$ clearance by internalization of $A \beta$ and efficiently eliminate $A \beta$ microaggregates from the brain into circulation [22]. Consequently, we examined the potential therapeutic effect of MDP in the APP mouse model of AD. In the first protocol (high frequency), behavioral test results show slight improvement (tendency) in memory function, whereas APP mice treated with MDP in the second protocol (low 
frequency) demonstrated significant improvement in the memory test. The memory test results obtained from both protocols suggest that the chronic administration of MDP at lower frequency is sufficient to delay the appearance of an Alzheimer-like phenotype. Whether other factor(s) in the two protocols besides the treatment frequency contributed to the difference in memory test results is an open question. In postmortem analysis, we first examined microglial activation and $A \beta$ levels. Nevertheless, we did not observe any change in $A \beta$ burden or microglial activation, suggesting that overall memory improvements observed in behavioral tests, especially low-frequency protocol, are dependent on other factor(s) involved in MDP-mediated cognitive improvement.

Previous reports demonstrated that the degree of synapse loss is a stronger correlate of cognitive decline in $\mathrm{AD}$ than counts and/or size of plaques [36-38]. We found that PSD95 protein expression level significantly increased in the APP mice treated with MDP compared to that of control. PSD95 is the most abundant protein in the excitatory postsynaptic density. Furthermore, PSD95 is a master regulator of neuronal plasticity and memory [39] and has previously been shown to be decreased in the APP mouse model of AD [40]. Interestingly, other studies demonstrated the role of PSD95 in interacting and regulating adhesion molecules, signaling proteins, scaffolding proteins, and cytoskeletal proteins $[41,42]$. It is then possible that MDP-mediated PSD95 regulation is beyond its role in stabilizing the neuronal circuitry.

PSD95 also interacts and co-localizes with LRP1 [43, 44]. Interestingly, the LRP1 protein expression levels also increased significantly in the group treated with MDP. Accumulating evidence also suggests that LRP1 is a key player in $\mathrm{AD}$ pathology at the $\mathrm{BBB}$ level [45]. Indeed, LRP1 is involved not only in $A \beta$ endocytosis and cerebral degradation mediated by neurons, but it is also a key player to eliminate $A \beta$ across the $B B B[35,46,47]$. In parallel, we observed a significant increase in COX2 expression levels in the MDP-treated group. While excessive COX2 expression plays a key role in neuroinflammation [48], several studies clearly showed that both constitutive and inducible COX2 play an important role in the refinement of synaptic activity [32, 49]. More importantly, the involvement of COX2 in long-term synaptic plasticity and cognition has been supported by several behavioral tests, reviewed by [50]. Similar to previous reports, we did not observe a significant increase in inflammatory marker expression levels in the brain, but we found a modulation of COX2 expression levels. In the context of MDP treatment, future studies are needed to confirm the positive effect of COX2 on the synaptic function that has been reported previously.
Taken together, considering these results, it becomes tempting to suggest that PSD95 and LRP1 are two key factors involved in MDP-derived memory improvement via enhancement of synapse function and vascular $A \beta$ clearance. In addition to the role of LRP1 in vascular A $\beta$ clearance and considering the upregulation of PSD95, it is tempting to propose a role of LRP1 as an endocytic receptor for the neuronal clearance of $A \beta$. This needs to be further investigated to understand the differential roles of LRP1 in A $\beta$ clearance in the context of MDP mediating immune regulation (Fig. 5).

To further confirm the contribution of peripheral monocyte recruitments in vascular $A \beta$ clearance, we assessed MCP-1 expression levels. Previous reports from our group demonstrated MCP-1-mediated monocyte recruitment in the brain vascular system [51]. MCP-1 protein expression levels increased significantly in APP mice treated with MDP compared to controls. Interestingly, we found no significant change in the protein expression level of the NF-kB. Since NF-kB is an inflammatory mediator involved in MCP-1 production, we believe the increase in MCP-1 expression level may not be dependent on the pro-inflammatory response. To further explore this phenomenon, we next analyzed the endothelial inflammatory biomarkers, VCAM-1, and ICAM-1 (Chakraborty, De Wit, et al. 2017). While VACM-1 showed no significant changes, we observed a significant reduction in ICAM-1 protein expression level in APP mice treated with MDP compared to controls. Consistent with our previous observations, these results indicate that MDP treatments favor chemotactic gradients to allow the recruitment of monocytes/macrophages to the brain vascular system without being associated with neuroinflammation.

Using live intravital two-photon microscopy, we investigated whether MDP-mediated shifting towards Ly6 $\mathrm{C}^{\text {low }}$ monocytes could drive vascular $A \beta$ clearance via $A \beta$ uptake by Ly6 ${ }^{\text {low }}$ monocytes (data not shown). We must confirm the data using more mice per group (we have used 1 mouse/group). Nevertheless, based on our preliminary data, crawling $\mathrm{GFP}^{+}$cells in $\mathrm{APP}_{\text {swe }} / \mathrm{PS}^{+/-}$/ $\mathrm{Cx} 3 \mathrm{CR} 1^{\mathrm{GFP} /+}$ mouse appeared to be more frequent in blood vessels containing small $A \beta$ aggregates when treated with MDP compared with the vehicle one. Interestingly, following MDP treatment in APP and WT mice, the $\mathrm{GFP}^{+}$cells seem to be more frequent in the APP mouse with blood vessels containing small $A \beta$ aggregates, but not in the WT mouse (data not shown).

This study also raises questions about the effect of MDP on microglial activation and function. We identified no difference between Iba1 protein levels, suggesting that MDP might regulate only systemic myeloid cells, mainly Ly6C $C^{\text {high }}$ and Ly6C ${ }^{\text {low }}$ monocytes. However, this does not rule out an indirect effect of MDP on the activity of microglia. Future studies using different time 


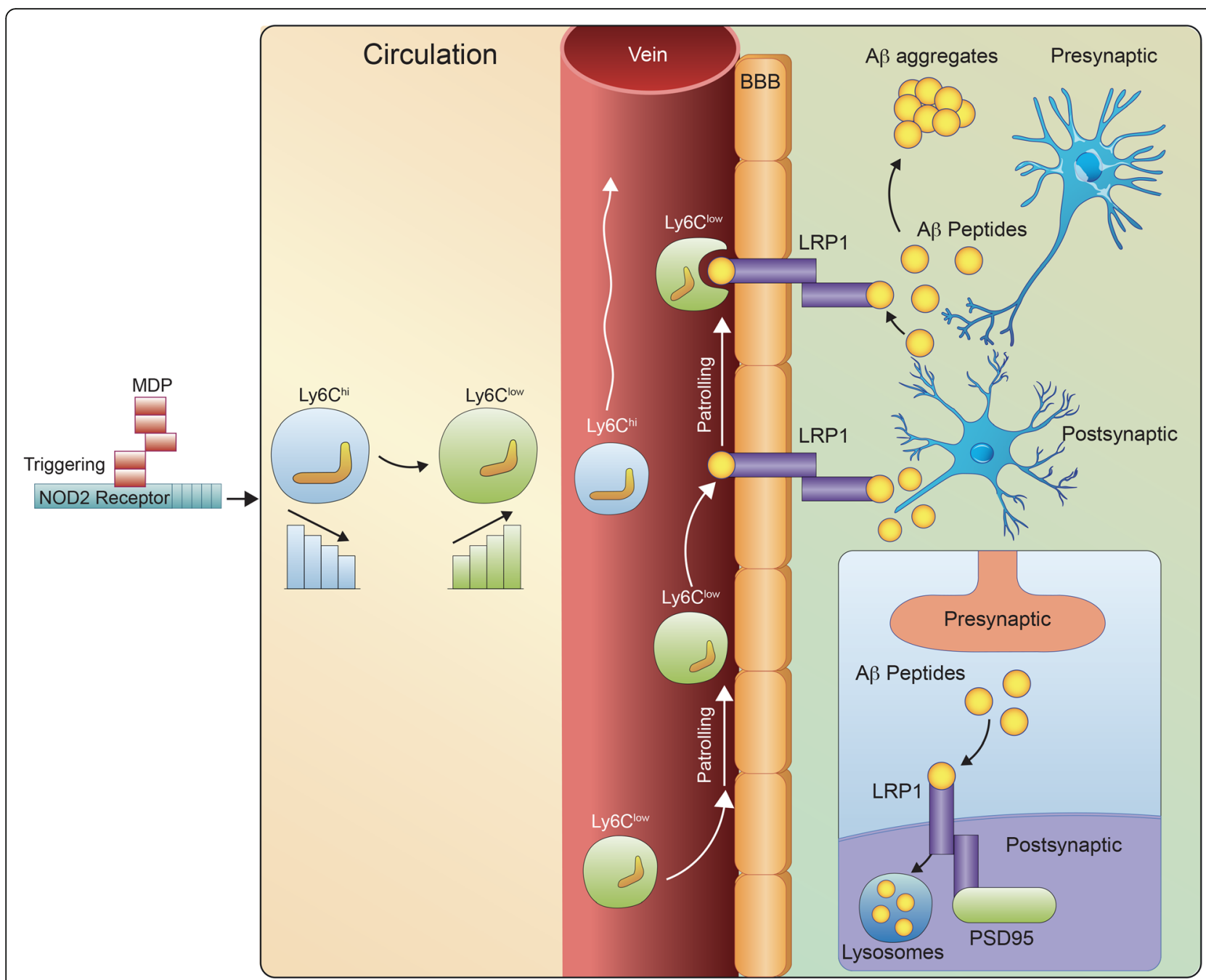

Fig. 5 A scheme summarizing MDP-mediated immunomodulatory effects on the APP mouse model of AD via converting inflammatory monocytes into patrolling monocytes. MDP treatments improved cognitive declines and A $\beta$ clearance in APP mice and increased expression levels of PSD95 and LRP1 receptors

points after MDP administrations in different disease stages are needed to determine possible indirect effects of MDP, specifically on microglia.

\section{Conclusions}

Our findings demonstrate selective immunomodulatory effects of MDP on a mouse model of AD. Medications that solely target specific monocyte subsets and monocyte-derived macrophages with mild immunomodulatory effects in neurodegenerative disease, without triggering microglial activation, are rare. Here, we have shown the therapeutic effects of MDP administration in an APP mouse model of AD. Furthermore, we have provided solid evidence indicating the potential of MDP in terms of maintaining its therapeutic effect via regulating monocyte subsets in long-term administration (both in WT and APP model). Taken together, our results suggest that MDP may be beneficial in the early phase and, to some extent, late phase of AD.

\section{Supplementary information}

Supplementary information accompanies this paper at https://doi.org/10. 1186/s12974-020-01893-3.

Additional file 1. Microglial activation and $A \beta$ burden analysis. Flow cytometry protocols. Details of microglial activation and $A \beta$ load analysis, and flow cytometry protocols for extracellular staining as well as gating strategy

\section{Abbreviations}

AD: Alzheimer's disease; APP: Amyloid precursor protein; AB: Amyloid-beta; BSA: Bovine serum albumin; CAA: Cerebral amyloid angiopathy;

COX2: Cyclooxygenase-II; DAB: 3,3'-Diaminobenzidine; DAPI: 4',6-diamidino-2phenylindole; EDTA: Ethylene diamine tetraacetic acid; ICAM: Intercellular Adhesion Molecule; KPBS: Phosphate-buffered saline containing potassium; MCP-1: monocyte chemoattractant protein-1; MDP: Muramyl dipeptide; NGS: Normal goat serum; PS1: Presenilin 1; TREM2: Triggering receptor 
expressed on myeloid cells 2; VCAM: Vascular cell adhesion protein; WT: Wild type

\section{Acknowledgements}

We thank Emilie Lambert-Cyr and Kassandra Gagnon for their useful help for immunoblot experiments.

\section{Authors' contributions}

AFM participated in the design of experiments, conducted the animal protocols and experiments, analyzed and interpreted the data, wrote the manuscript, and assembled the figures. GC contributed to the design of the experimental protocols, supervised and conducted the experiments and analyses with the Alzheimer model, and revised the manuscript and figures. MMP performed immunoblot and part of histological experiments. PP performed Two-photon microscopy experiment and assembled the related figure panel. NL helped for stereological analyses and helped to maintain mice colonies. JG helped to formulate the study concept. SR formulated the study concept and all experimental designs, supervised the project, and wrote and revised the manuscript deeply. The authors read and approved the final manuscript.

\section{Funding}

This work was supported by the Canadian Institutes in Health Research (CIHR) via the foundation scheme program. SR is supported by a Canadian Research Chair in Neuroimmunology.

\section{Availability of data and materials}

The data supporting the findings of this study are available from the corresponding author upon request.

\section{Ethics approval and consent to participate}

All protocols were performed according to the Canadian Council on Animal Care guidelines, as administered by the Laval University Animal Welfare Committee. All experiments were approved by the local committee.

\section{Consent for publication}

Not applicable.

\section{Competing interests}

The authors declare that they have no competing interests.

\section{Author details}

${ }^{1}$ Neuroscience Laboratory, CHU de Quebec Research Center and Department of Molecular Medicine, Faculty of Medicine, Laval University, 2705 Laurier Boulevard, Quebec City, QC G1V 4G2, Canada. ${ }^{2}$ Laboratory of Innate Immunity, CHU of Quebec Research Center and Department of Molecular Medicine, Faculty of Medicine, Laval University, 2705 Laurier Boulevard, Quebec City, QC G1V 4G2, Canada.

Received: 26 February 2020 Accepted: 13 July 2020

Published online: 22 July 2020

\section{References}

1. Girardin SE, Boneca IG, Viala J, Chamaillard M, Labigne A, Thomas G, et al. Nod2 is a general sensor of peptidoglycan through muramyl dipeptide (MDP) detection. J Biol Chem. 2003;278(11):8869-72.

2. Grimes $\mathrm{CL}$, Ariyananda LDZ, Melnyk JE, O'Shea EK. The innate immune protein Nod2 binds directly to MDP, a bacterial cell wall fragment. J Am Chem Soc. 2012;134(33):13535-7.

3. Ogura Y, Bonen DK, Inohara N, Nicolae DL, Chen FF, Ramos R, et al. A frameshift mutation in NOD2 associated with susceptibility to Crohn's disease. Nature. 2001:411(6837):603.

4. Auffray C, Sieweke MH, Geissmann F. Blood monocytes: development, heterogeneity, and relationship with dendritic cells. Annu Rev Immunol. 2009;27.

5. Guilliams M, Mildner A, Yona S. Developmental and functional heterogeneity of monocytes. Immunity. 2018;49(4):595-613.

6. Geissmann F, Jung S, Littman DR. Blood monocytes consist of two principal subsets with distinct migratory properties. Immunity. 2003;19(1):71-82.
7. Jakubzick C, Gautier EL, Gibbings SL, Sojka DK, Schlitzer A, Johnson TE, et al. Minimal differentiation of classical monocytes as they survey steady-state tissues and transport antigen to lymph nodes. Immunity. 2013;39(3):599-610.

8. Palframan RT, Jung $S$, Cheng G, Weninger W, Luo Y, Dorf M, et al. Inflammatory chemokine transport and presentation in HEV: a remote control mechanism for monocyte recruitment to lymph nodes in inflamed tissues. J Exp Med. 2001;194(9):1361-74.

9. Jung S, Aliberti J, Graemmel P, Sunshine MJ, Kreutzberg GW, Sher A, et al. Analysis of fractalkine receptor CX3CR1 function by targeted deletion and green fluorescent protein reporter gene insertion. Mol Cell Biol. 2000;20(11):4106-14.

10. Naert G, Rivest S. A deficiency in CCR2+ monocytes: the hidden side of Alzheimer's disease. J Mol Cell Biol. 2013;5(5):284-93.

11. Haass C, Selkoe DJ. Soluble protein oligomers in neurodegeneration: lessons from the Alzheimer's amyloid $\beta$-peptide. Nat Rev Mol Cell Biol. 2007;8(2):101.

12. Jack CR Jr, Knopman DS, Jagust WJ, Petersen RC, Weiner MW, Aisen PS, et al. Tracking pathophysiological processes in Alzheimer's disease: an updated hypothetical model of dynamic biomarkers. Lancet Neurology. 2013;12(2):207-16

13. Pimentel-Coelho PM, Rivest S. The early contribution of cerebrovascular factors to the pathogenesis of Alzheimer's disease. Eur J Neurosci. 2012; 35(12):1917-37.

14. Weller RO, Preston SD, Subash M, Carare RO. Cerebral amyloid angiopathy in the aetiology and immunotherapy of Alzheimer disease. Alzheimers Res Ther. 2009;1(2):6.

15. Deane R, Bell R, Sagare A, Zlokovic B. Clearance of amyloid- $\beta$ peptide across the blood-brain barrier: implication for therapies in Alzheimer's disease. CNS Neurol Disord Drug Targets. 2009;8(1):16-30.

16. Marques MA, Kulstad JJ, Savard CE, Green PS, Lee SP, Craft S, et al. Peripheral amyloid- $\beta$ levels regulate amyloid- $\beta$ clearance from the central nervous system. J Alzheimers Dis. 2009;16(2):325-9.

17. Sehgal N, Gupta A, Valli RK, Joshi SD, Mills JT, Hamel E, et al. Withania somnifera reverses Alzheimer's disease pathology by enhancing low-density lipoprotein receptor-related protein in liver. Proc Natl Acad Sci U S A. 2012; 109(9):3510-5.

18. Ransohoff RM, Kivisäkk P, Kidd G. Three or more routes for leukocyte migration into the central nervous system. Nat Rev Immunol. 2003;3(7):569.

19. Hawkes CA, McLaurin J. Selective targeting of perivascular macrophages for clearance of $\beta$-amyloid in cerebral amyloid angiopathy. Proc Natl Acad Sci U S A. 2009;106(4):1261-6.

20. Mildner A, Schlevogt B, Kierdorf K, Böttcher C, Erny D, Kummer MP, et al. Distinct and non-redundant roles of microglia and myeloid subsets in mouse models of Alzheimer's disease. J Neurosci. 2011;31(31):11159-71.

21. Saresella M, Marventano I, Calabrese E, Piancone F, Rainone V, Gatti A, et al. A complex proinflammatory role for peripheral monocytes in Alzheimer's disease. J Alzheimers Dis. 2014;38(2):403-13.

22. Michaud J-P, Bellavance M-A, Préfontaine $P$, Rivest $S$. Real-time in vivo imaging reveals the ability of monocytes to clear vascular amyloid beta. Cell Rep. 2013;5(3):646-53.

23. Lampron A, Larochelle A, Laflamme N, Préfontaine P, Plante M-M, Sánchez $M G$, et al. Inefficient clearance of myelin debris by microglia impairs remyelinating processes. J Exp Med. 2015;212(4):481-95.

24. Lessard $A-J$, LeBel $M$, Egarnes $B$, Préfontaine $P$, Thériault $P$, Droit $A$, et al. Triggering of NOD2 receptor converts inflammatory Ly6C high into Ly6C low monocytes with patrolling properties. Cell Rep. 2017;20(8):1830-43.

25. Laflamme N, Cisbani $G$, Préfontaine $P$, Srour $Y$, Bernier J, St-Pierre $M-K$, et al. mCSF-induced microglial activation prevents myelin loss and promotes its repair in a mouse model of multiple sclerosis. Front Cell Neurosci. 2018;12:178.

26. Thériault P, ElAli A, Rivest S. High fat diet exacerbates Alzheimer's diseaserelated pathology in APPswe/PS1 mice. Oncotarget. 2016;7(42):67808.

27. Michaud J-P, Hallé $M$, Lampron $A$, Thériault $P$, Préfontaine $P$, Filali $M$, et al. Toll-like receptor 4 stimulation with the detoxified ligand monophosphoryl lipid A improves Alzheimer's disease-related pathology. Proc Natl Acad Sci U S A. 2013;110(5):1941-6.

28. Hui CW, St-Pierre M-K, Detuncq J, Aumailley L, Dubois M-J, Couture V, et al. Nonfunctional mutant Wrn protein leads to neurological deficits, neuronal stress, microglial alteration, and immune imbalance in a mouse model of Werner syndrome. Brain Behav Immun. 2018;73:450-69.

29. Guariglia SR, Chadman KK. Water T-maze: a useful assay for determination of repetitive behaviors in mice. J Neurosci Methods. 2013;220(1):24-9.

30. Sharma S, Rakoczy S, Brown-Borg H. Assessment of spatial memory in mice. Life Sci. 2010;87(17-18):521-36. 
31. Scheuner D, Eckman C, Jensen M, Song X, Citron M, Suzuki N, et al. Secreted amyloid $\beta$-protein similar to that in the senile plaques of Alzheimer's disease is increased in vivo by the presenilin 1 and 2 and APP mutations linked to familial Alzheimer's disease. Nat Med. 1996;2(8):864.

32. Sang N, Chen C. Lipid signaling and synaptic plasticity. Neuroscientist. 2006; 12(5):425-34.

33. Hölscher C. Inhibitors of cyclooxygenases produce amnesia for a passive avoidance task in the chick. Eur J Neurosci. 1995;7(6):1360-5.

34. Sato T, Ishida T, Irifune M, Tanaka K-i, Hirate K, Nakamura N, et al. Effect of NC-1900, an active fragment analog of arginine vasopressin, and inhibitors of arachidonic acid metabolism on performance of a passive avoidance task in mice. Eur J Pharmacol. 2007;560(1):36-41.

35. Kanekiyo T, Cirrito JR, Liu C-C, Shinohara M, Li J, Schuler DR, et al. Neuronal clearance of amyloid- $\beta$ by endocytic receptor LRP1. J Neurosci. 2013;33(49): 19276-83.

36. DeKosky ST, Scheff SW. Synapse loss in frontal cortex biopsies in Alzheimer's disease: correlation with cognitive severity. Ann Neurol. 1990;27(5):457-64

37. Terry RD, Masliah E, Salmon DP, Butters N, DeTeresa R, Hill R, et al. Physical basis of cognitive alterations in Alzheimer's disease: synapse loss is the major correlate of cognitive impairment. Ann Neurol. 1991;30(4):572-80.

38. Hong S, Beja-Glasser VF, Nfonoyim BM, Frouin A, Li S, Ramakrishnan S, et al. Complement and microglia mediate early synapse loss in Alzheimer mouse models. Science. 2016;352(6286):712-6.

39. Bustos FJ, Ampuero E, Jury N, Aguilar R, Falahi F, Toledo J, et al. Epigenetic editing of the Dlg4/PSD95 gene improves cognition in aged and Alzheimer's disease mice. Brain. 2017;140(12):3252-68.

40. Hou Y, Aboukhatwa MA, Lei D-L, Manaye K, Khan I, Luo Y. Anti-depressant natural flavonols modulate BDNF and beta amyloid in neurons and hippocampus of double TgAD mice. Neuropharmacology. 2010;58(6):911-20.

41. van Zundert B, Yoshii A, Constantine-Paton M. Receptor compartmentalization and trafficking at glutamate synapses: a developmental proposal. Trends Neurosci. 2004;27(7):428-37.

42. Elias GM, Nicoll RA. Synaptic trafficking of glutamate receptors by MAGUK scaffolding proteins. Trends Cell Biol. 2007;17(7):343-52.

43. Niethammer $\mathrm{M}$, Kim $\mathrm{E}$, Sheng $\mathrm{M}$. Interaction between the $\mathrm{C}$ terminus of NMDA receptor subunits and multiple members of the PSD-95 family of membrane-associated guanylate kinases. J Neurosci. 1996;16(7):2157-63.

44. Martin AM, Kuhlmann C, Trossbach S, Jaeger S, Waldron E, Roebroek A, et al. The functional role of the second NPXY motif of the LRP1 $\beta$-chain in tissuetype plasminogen activator-mediated activation of $\mathrm{N}$-methyl-D-aspartate receptors. J Biol Chem. 2008;283(18):12004-13.

45. Storck SE, Meister S, Nahrath J, Meißner JN, Schubert N, Di Spiezio A, et al. Endothelial LRP1 transports amyloid- $\beta 1-42$ across the blood-brain barrier. J Clin Invest. 2016;126(1):123-36.

46. Nazer B, Hong S, Selkoe DJ. LRP promotes endocytosis and degradation, but not transcytosis, of the amyloid- $\beta$ peptide in a blood-brain barrier in vitro model. Neurobiol Dis. 2008;30(1):94-102.

47. Kanekiyo T, Liu C-C, Shinohara M, Li J, Bu G. LRP1 in brain vascular smooth muscle cells mediates local clearance of Alzheimer's amyloid- $\beta$. J Neurosci. 2012;32(46):16458-65.

48. Minghetti L. Cyclooxygenase-2 (COX-2) in inflammatory and degenerative brain diseases. J Neuropathol Exp Neurol. 2004;63(9):901-10.

49. Bosetti F, Langenbach R, Weerasinghe GR. Prostaglandin E2 and microsomal prostaglandin E synthase-2 expression are decreased in the cyclooxygenase2-deficient mouse brain despite compensatory induction of cyclooxygenase-1 and Ca2 + -dependent phospholipase A2. J Neurochem. 2004;91(6):1389-97.

50. Yang $\mathrm{H}$, Chen C. Cyclooxygenase-2 in synaptic signaling. Curr Pharm Des. 2008:14(14):1443-51.

51. AR Simard, et al. Bone marrow-derived microglia play a critical role in restricting senile plaque formation in Alzheimer's disease. Neuron. 2006; 49(4):489-502

\section{Publisher's Note}

Springer Nature remains neutral with regard to jurisdictional claims in published maps and institutional affiliations.

\section{Ready to submit your research? Choose BMC and benefit from:}

- fast, convenient online submission

- thorough peer review by experienced researchers in your field

- rapid publication on acceptance

- support for research data, including large and complex data types

- gold Open Access which fosters wider collaboration and increased citations

- maximum visibility for your research: over $100 \mathrm{M}$ website views per year

At BMC, research is always in progress.

Learn more biomedcentral.com/submissions 